\title{
Sliding Mode Control of a Pneumatic Haptic Teleoperation System with on/off Solenoid Valves
}

\author{
M.Q. Le, M.T. Pham, M. Tavakoli, R. Moreau
}

\begin{abstract}
This paper presents a novel bilateral control scheme for pneumatic teleoperation systems that are actuated by lowcost solenoid valves. A sliding mode control is incorporated into a two-channel, bilateral teleoperation architecture involving position-position, force-force, or force-position schemes. An analysis of stability and transparency of the closed-loop teleoperation system is carried out. The proposed control design is verified on a single-degree-of-freedom pneumatic teleoperation system with four on/off solenoid valves. Moreover, simulation results demonstrate high accuracies in terms of position and force tracking in the teleoperation system.

Index terms-Pneumatic actuator, on/off solenoid valve, sliding mode control, haptic teleoperation, stability.
\end{abstract}

\section{INTRODUCTION}

Teleoperators are designed to enable humans to manipulate dangerous, remote, or delicate tasks with enhanced safety, at lower cost, or even with better accuracy than direct manipulation. Teleoperation has found applications in many areas including space and underwater explorations, nuclear waste handling, surgery, rehabilitation, training, education, and entertainment [1], [2].

In a teleoperation system, the human operator imposes a force on the master manipulator which results in a displacement that is transmitted to the slave [3]. When the slave follows the master's motion and the force interaction with the environment is driven to the operator, the teleoperation system is said to be bilateral. The goal of bilateral teleoperation is to achieve "transparency", meaning that through the master manipulator, the operator feels as if he/she is directly operating on the remote environment [4].

In this study, we investigate the use of electro-pneumatic actuators in a teleoperation system. Compared to the electrical actuators, which are most used today in teleoperation, pneumatic actuators have higher power-tomass ratio and can generate larger force without any reduction mechanism [5]. Moreover, they are inert to magnetic fields, which is crucial in certain applications such as robot-assisted surgery under MRI guidance [6].

In most recent work involving pneumatic actuation, servo-valves, rather than solenoid (on/off) valves, have been used to achieve high performances in various position or force control tasks. Servo-valves, however, are typically

M.Q. LE, M.T. Pham, and R. Moreau are with the Laboratoire Ampère, UMR CNRS 5005, Université de Lyon, INSA-Lyon, France (corresponding author's e-mail: minh-quyen.le@insa-lyon.fr).

M. Tavakoli is with the Department of Electrical and Computer Engineering, University of Alberta, Canada. expensive due to requiring high-precision manufacturing. Therefore, in this paper, fast-switching on/off valves are used due to their advantages in terms of low cost and small size. One of the objectives of this paper is to show that good transparency can be obtained with these inexpensive components as actuators of the teleoperation system.

The traditional control approach for systems with solenoid valves involves using Pulse Width Modulation (PWM) to control the output mass flow rate of the valve [79]. A main disadvantage of the PWM control is the chattering phenomenon caused by the high-frequency switching of the valve in steady state [10], [11].

To overcome the drawbacks of PWM-based control of solenoid valves, this paper presents a nonlinear sliding mode control inspired by Nguyen et al. [12]. The sliding control law is used in a two-channel $(2 \mathrm{CH})$ bilateral teleoperation architecture with three different schemes: position-position, force-force, and force-position. These architectures are chosen due to their simplicity in terms of implementation of the control law. We provide in this paper both a tracking performance analysis and a stability analysis for the closedloop system using a Lyapunov candidate function.

For the sake of simplicity, the master and slave actuators are considered to be identical in this study - indeed, the master and the slave are one degree-of-freedom (DOF) pneumatic manipulators. It should be noted that this paper does not deal with the problem of compensating for time delays in a teleoperation system's communication channel.

The structure of this paper is described as follows. The modeling of the pneumatic manipulator is presented in Section II. Section III describes the sliding controller design for a $2 \mathrm{CH}$ master-slave telemanipulator. Section IV presents simulation results that validate the proposed control laws. Finally, concluding remarks and perspectives are given in Section V.

\section{MODEL OF THE PNEUMATIC SYSTEM}

As mentioned above, the master and the slave manipulators are identical, thus only one pneumatic robot is presented in this section. To describe the air flow dynamics in a cylinder, we assume that air is a perfect gas, the mass flow rate leakages are negligible, and the temperature variation in chambers is negligible with respect to the supply temperature. A schematic of the one-DOF pneumatic actuation system is shown in Fig. 1. The device consists of a pneumatic cylinder, four solenoid valves, a force sensor, and a position sensor. 


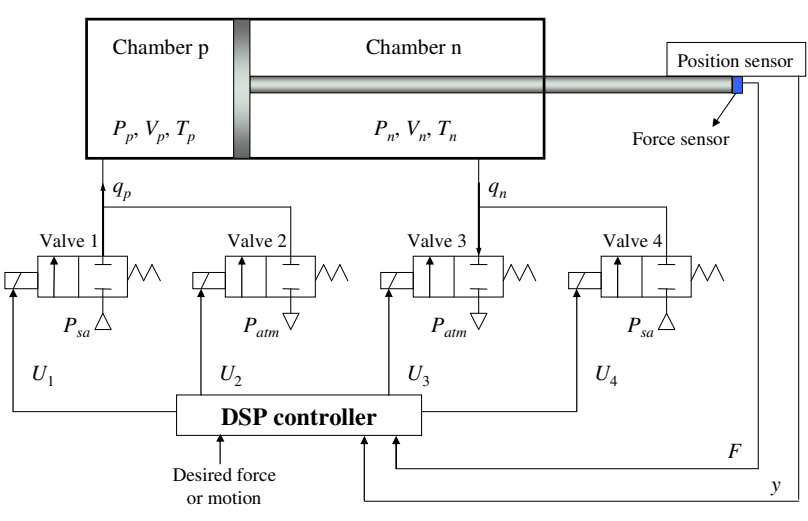

Fig. 1. Electro-pneumatic system with four valves

The behavior of the pressure inside each chamber of the cylinder can be expressed as [13]

$$
\begin{aligned}
& \dot{P}_{p}=\frac{\gamma r T_{a}}{V_{p}(y)}\left(q_{p}\left(U_{1}, U_{2}, P_{p}\right)-\frac{P_{p}}{r T_{a}} S_{p} \dot{y}\right) \\
& \dot{P}_{n}=\frac{\gamma r T_{a}}{V_{n}(y)}\left(q_{n}\left(U_{3}, U_{4}, P_{n}\right)+\frac{P_{n}}{r T_{a}} S_{n} \dot{y}\right)
\end{aligned}
$$

where $U_{1}, U_{2}, U_{3}$ and $U_{4}$ are the control voltages of valve 1 , valve 2 , valve 3 , and valve $4 ; y$ and $\dot{y}$ are the position (m) and velocity $(\mathrm{m} / \mathrm{s})$ of the piston; $P_{p}$ and $P_{n}$ are the pressures inside chambers $\mathrm{p}$ and $\mathrm{n}(\mathrm{Pa}) ; V_{p}$ and $V_{n}$ are the volumes of chambers $\mathrm{p}$ and $\mathrm{n}\left(\mathrm{m}^{3}\right) ; S_{p}$ and $S_{n}$ are the piston cylinder area in the chambers $\mathrm{p}$ and $\mathrm{n}\left(\mathrm{m}^{2}\right) ; q_{p}$ and $q_{n}$ are the mass flow rates in the chambers $\mathrm{p}$ and $\mathrm{n}(\mathrm{kg} / \mathrm{s}) ; T_{a}$ is the temperature of the supply air $(\mathrm{K}) ; r$ is the perfect gas constant $(\mathrm{J} /(\mathrm{kg} . \mathrm{K}))$ and $\gamma$ is the polytropic constant.

The mass flow rate characteristics of the on/off valves can be expressed as

$$
\begin{aligned}
& q_{p}\left(U_{1}, U_{2}, P_{p}\right)=\left\{\begin{array}{ccc}
q\left(P_{s a}, P_{p}\right) & \text { for } & U_{1}=1 \text { and } U_{2}=0 \\
0 & \text { for } & U_{1}=0 \text { and } U_{2}=0 \\
-q\left(P_{p}, P_{a t m}\right) & \text { for } & U_{1}=0 \text { and } U_{2}=1
\end{array}\right. \\
& q_{n}\left(U_{3}, U_{4}, P_{n}\right)=\left\{\begin{array}{ccc}
q\left(P_{s a}, P_{n}\right) & \text { for } & U_{3}=0 \text { and } U_{4}=1 \\
0 & \text { for } & U_{3}=0 \text { and } U_{4}=0 \\
-q\left(P_{n}, P_{a t m}\right) & \text { for } & U_{3}=1 \text { and } U_{4}=0
\end{array}\right.
\end{aligned}
$$

where $P_{s a}$ and $P_{a t m}$ are the pressures of the supply air and atmosphere. The ' 0 ' state of the input voltage corresponds to a closed valve and the ' 1 ' state corresponds to an open valve. All the states where $U_{1}=U_{2}=1$ and $U_{3}=U_{4}=1$ are prohibited to avoid a bypass of the valves. The functions in (2) are given by a standard expression in which the mass flow rate of the valve is regulated by the air passage through an orifice [14]:

$$
q\left(P_{u p}, P_{\text {down }}\right)= \begin{cases}C_{v a l} P_{u p} \sqrt{\frac{T_{a t m}}{T_{u p}}} & \text { if } \frac{P_{\text {down }}}{P_{u p}} \leq 0.433 \text { (sonic) } \\ C_{v a l} P_{u p} \sqrt{\frac{T_{a t m}}{T_{u p}}} \sqrt{1-\left(\frac{\frac{P_{d o w n}}{P_{u p}}-C_{r}}{1-C_{r}}\right)^{2}} & \text { otherwise (subsonic) }\end{cases}
$$

In the above, $C_{v a l}$ is the valve flow rate coefficient; $P_{u p}$ and $P_{\text {down }}$ are respectively the absolute upstream and downstream stagnation pressures of the valve $(\mathrm{Pa}) ; T_{a t m}$ is the atmosphere temperature, and $T_{u p}$ is the upstream stagnation temperature.

The dynamics of the piston and the load are

$$
M \ddot{y}=S_{p} P_{p}-S_{n} P_{n}-b \dot{y}-F_{s t}+F_{e x t}
$$

where $b$ is the viscous friction coefficient (N.s/m); $M$ is the moving load $(\mathrm{kg}) ; F_{s t}$ is the stiction force; and $F_{\text {ext }}$ is the external force $(\mathrm{N})$. For the sake of simplicity, the stiction force is supposed to be negligible.

\section{TELEOPERATION BASED ON SLIDING CONTROL}

In order to facilitate the control law design, a switching scheme for the four solenoid valves in Fig. 1 is defined so that each of the master and slave robots has the three modes of operation shown in Table I

\begin{tabular}{cccc}
\hline \multicolumn{4}{c}{ TABLE I: THREE POSSIBLE CONTROL MODES } \\
\hline Mode 1 & Mode 2 & Mode 3 \\
\hline Chamber $\mathrm{p}$ & filled & exhausted & closes \\
\hline Chamber $\mathrm{n}$ & exhausted & filled & closes \\
\hline Control $u$ & 1 & -1 & 0 \\
\hline$U=\left[\begin{array}{llll}U_{1} & U_{2} & U_{3} & U_{4}\end{array}\right]$ & {$\left[\begin{array}{llll}1 & 0 & 1 & 0\end{array}\right]$} & {$\left[\begin{array}{llll}0 & 1 & 0 & 1\end{array}\right]$} & {$\left[\begin{array}{lll}0 & 0 & 0\end{array}\right]$} \\
\hline
\end{tabular}

Here, $U$ (the $4^{\text {th }}$ row) is defined as the input vector of the valves, and $u$ (the $3^{\text {rd }}$ row) is the discrete (switching) control can be chosen either as $u=\operatorname{sign}(s)$ or $u=-\operatorname{sign}(s)$ where $s$ is the sliding surface.

In order to bring the system to the sliding surface $s=0$ at steady state, we define a neighbourhood of radius $\varepsilon<<1$ around zero. When $|s|$ is within this neighbourhood, the third mode $(u=0)$ is used to save energy. In summary, we get the control law as

$$
u=\left\{\begin{array}{lll}
+/-\operatorname{sign}(s) & \text { if } & |s|>\varepsilon \\
0 & \text { if } & |s| \leq \varepsilon
\end{array}\right.
$$

\section{A. Open-loop models of master and slave}

Ignoring the stiction force in (4), the dynamics of the master and slave robots can be written as

$$
\begin{aligned}
& M \ddot{y}_{m}=S_{p} P_{p, m}-S_{n} P_{n, m}-b \dot{y}_{m}+f_{h} \\
& M \ddot{y}_{s}=S_{p} P_{p, s}-S_{n} P_{n, s}-b \dot{y}_{s}-f_{e}
\end{aligned}
$$

where $f_{h}$ and $f_{e}$ are the operator force exerted on the master and the environment force exerted on the slave; $y_{m}$ and $y_{s}$ are the master and slave positions.

Differentiating (6) and using (1)-(3), the dynamics of the master and slave manipulators are obtained after some manipulations as [12]

$\dddot{y}_{m}=\left\{\begin{array}{l}\alpha_{m}+\beta_{m}^{+}+\dot{f}_{h} / M, u_{m}=1 \\ \alpha_{m}-\beta_{m}^{-}+\dot{f}_{h} / M, u_{m}=-1, \dddot{y}_{s} \\ \alpha_{m}+\dot{f}_{h} / M \quad, u_{m}=0\end{array}=\left\{\begin{array}{l}\alpha_{s}+\beta_{s}^{+}-\dot{f}_{e} / M, u_{s}=1 \\ \alpha_{s}-\beta_{s}^{-}-\dot{f}_{e} / M, u_{s}=-1 \\ \alpha_{s}-\dot{f}_{e} / M \quad, u_{s}=0\end{array}\right.\right.$

where $i=m$ or $s$ (for master or slave, respectively), and 


$$
\begin{aligned}
& \alpha_{i}=-\frac{b}{M} \ddot{y}_{i}-\frac{\gamma}{M}\left(\frac{S_{p}^{2} P_{p, i}}{V_{p}\left(y_{i}\right)}+\frac{S_{n}^{2} P_{n, i}}{V_{n}\left(y_{i}\right)}\right) \dot{y}_{i} \\
& \beta_{i}^{+}=\gamma r T_{a}\left(\frac{S_{p}}{M} \frac{q\left(P_{s a}, P_{p, i}\right)}{V_{p}\left(y_{i}\right)}+\frac{S_{n}}{M} \frac{q\left(P_{n, i}, P_{a t m}\right)}{V_{n}\left(y_{i}\right)}\right) \\
& \beta_{i}^{-}=\gamma r T_{a}\left(\frac{S_{p}}{M} \frac{q\left(P_{p, i}, P_{a t m}\right)}{V_{p}\left(y_{i}\right)}+\frac{S_{n}}{M} \frac{q\left(P_{s a}, P_{n, i}\right)}{V_{n}\left(y_{i}\right)}\right)
\end{aligned}
$$

\section{B. Closed-loop teleoperation system}

\section{1) Position error based (PEB)}

A position-error-based, also called position-position, system involves the simplest bilateral controller in which no force sensors are required. This architecture involves two types of data transmission between the master and the slave: position from the master to the slave and vice versa. The pneumatic-actuated PEB teleoperation system with a sliding mode control is shown in Fig. 2.

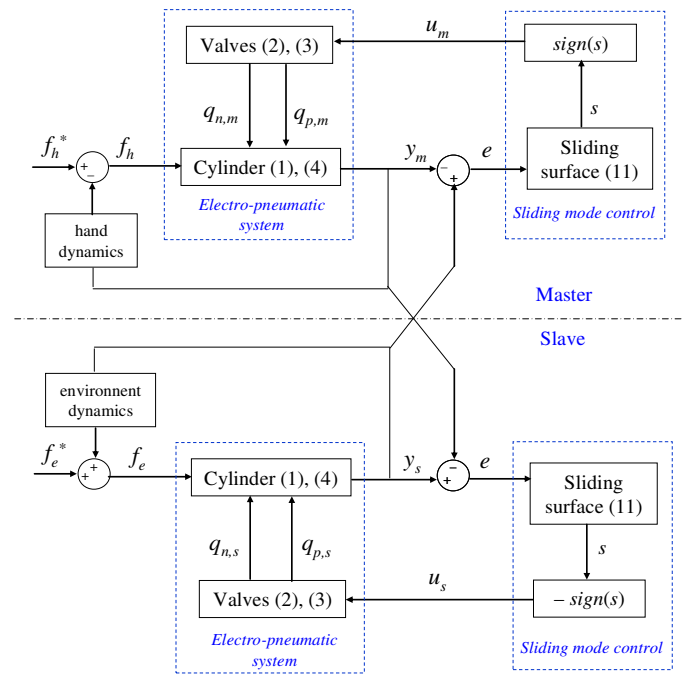

Fig. 2. Position-error-based approach with sliding mode control

In Fig. 2, $s$ is the sliding surface; $u_{m}$ and $u_{s}$ are the discrete control signals for the master and the slave manipulators; and $e=y_{s}-y_{m}$ is the position tracking error. Also, $f_{h}{ }^{*}$ and $f_{e}^{*}$ are the operator's and the environment's exogenous input forces, respectively.

In the PEB scheme, the sliding surface $s$ is defined as

$$
s=\ddot{e}+2 \xi \omega \dot{e}+\omega^{2} e
$$

where $e=y_{s}-y_{m}$ is the position error between the master and the slave and $\xi$ and $\omega$ are constant and positive parameters. The control laws $u_{m}$ and $u_{s}$ are defined as $u_{s}=-u_{m}=-$ $\operatorname{sign}(s)$.

In the following, we analyze the position error convergence and the stability of the closed-loop system.

Consider the following Lyapunov candidate function

$$
V=\frac{1}{2} s^{2}
$$

The sliding surface $s=0$ is reached within a finite time if the following condition is satisfied:

$$
\dot{V}=s \dot{s}<-\eta|s|
$$

for some constant $\eta>0$. Thus, from (11) and (13), we need

$$
s\left(\dddot{e}+2 \xi \omega \ddot{e}+\omega^{2} \dot{e}\right)<-\eta|s|
$$

Case 1: $s>0$. In this case, (14) becomes

$$
\left(\dddot{y}_{s}-\dddot{y}_{m}\right)+2 \xi \omega \ddot{e}+\omega^{2} \dot{e}<-\eta
$$

Since $s>0$, thus $u_{s}=-1$ and $u_{m}=1$. Therefore, the master and slave dynamics in (7) become

$$
\dddot{y}_{m}=\alpha_{m}+\beta_{m}^{+}+\dot{f}_{h} / M \quad, \quad \dddot{y}_{s}=\alpha_{s}-\beta_{s}^{-}-\dot{f}_{e} / M
$$

Substituting (16) in (15) results in the following condition

$$
\left(\alpha_{s}-\alpha_{m}\right)-\left(\beta_{m}^{+}+\beta_{s}^{-}\right)-\left(\dot{f}_{h}+\dot{f}_{e}\right) / M+2 \xi \omega \ddot{e}+\omega^{2} \dot{e}<-\eta
$$

In other words,

$$
\lambda-\left(\beta_{m}^{+}+\beta_{s}^{-}\right)<-\eta
$$

where $\lambda=\alpha_{s}-\alpha_{m}-\left(\dot{f}_{h}+\dot{f}_{e}\right) / M+2 \xi \omega \ddot{e}+\omega^{2} \dot{e}$

Case 2 : $s<0$. In this case, (14) becomes

$$
\left(\dddot{y}_{s}-\dddot{y}_{m}\right)+2 \xi \omega \ddot{e}+\omega^{2} \dot{e}>\eta
$$

Since $s<0$, then $u_{s}=1$ and $u_{m}=-1$. Hence, the master and slave dynamics in (7) can be expressed as

$$
\dddot{y}_{m}=\alpha_{m}-\beta_{m}^{-}+\dot{f}_{h} / M \quad, \quad \dddot{y}_{s}=\alpha_{s}+\beta_{s}^{+}-\dot{f}_{e} / M
$$

Substituting (20) in (15) yields

$$
\lambda+\left(\beta_{m}^{-}+\beta_{s}^{+}\right)>\eta
$$

Note that, from (9) and (10), $\beta_{i}^{+}$and $\beta_{i}^{-}$are positive, and can be made as large as desired by choosing a sufficiently large valve orifice $C_{v a l}$ in (3). Thus, to ensure that the conditions (18) and (21) (depending on the sign of $s$ ) are satisfied, we only need to show that $\lambda$ is bounded. For this, we utilize the dynamics of the operator and the environment

$$
\begin{aligned}
& f_{h}=-M_{h} \ddot{y}_{m}-B_{h} \dot{y}_{m}-K_{h} y_{m}+f_{h}^{*} \\
& f_{e}=M_{e} \ddot{y}_{s}+B_{e} \dot{y}_{m}+K_{e} y_{m}+f_{e}^{*}
\end{aligned}
$$

where $M_{h}, M_{e}, B_{h}, B_{e}, K_{h}$ and $K_{e}$ are assumed to be positive corresponding to the mass, damping and stiffness of the operator's hand and the environment, respectively.

Substituting (22) into (6) yields

$$
\begin{aligned}
& \left(M+M_{h}\right) \ddot{y}_{m}=S_{p} P_{p, m}-S_{n} P_{n, m}-\left(b+B_{h}\right) \dot{y}_{m}-K_{h} y_{m}+f_{h}^{*} \\
& \left(M+M_{e}\right) \ddot{y}_{s}=S_{p} P_{p, s}-S_{n} P_{n, s}-\left(b+B_{e}\right) y_{s}-K_{e} y_{s}-f_{e}^{*}
\end{aligned}
$$

To establish the boundedness of $\lambda$, we consider the following points:

- Since we are dealing with a physical system, the piston positions $y_{m}$ and $y_{s}$ are bounded at the beginning (i.e., at $t=0)$. Also, the interaction forces $f_{h}$ and $f_{e}$ are bounded at the beginning.

- In practice, the chamber pressures $P_{p, i}$ and $P_{n, i}$ are lowerbounded by the atmospheric pressure $\left(P_{a t m}\right)$ and upperbounded by the supply pressure $\left(P_{s a}\right)$. This means that the first-order differential equations (6) in terms of $\dot{y}_{m}$ and $\dot{y}_{s}$ leads to bounded solutions for the piston velocities. Since the signals in the right side of (6) are bounded, then $\ddot{y}_{m}$ and $\ddot{y}_{s}$ are also bounded. 
- The rate of change of pressures $\dot{P}_{p, i}$ and $\dot{P}_{n, i}$ are bounded because each relationship in (1) is defined by a mass flow rates, a velocity, a pressure and a chamber volume that are all bounded functions.

- The exogenous input forces $f_{h}{ }^{*}, f_{e}{ }^{*}$ and their derivatives are supposed to be bounded as they originate from the human operator and the environment. Hence, differentiating (23) yields the boundedness of $\dddot{y}_{m}$ and $\dddot{y}_{s}$. Consequently, by using the derivative of (22) we infer that $\dot{f}_{h}$ and $\dot{f}_{e}$ are also bounded.

Eventually, it is found that $\lambda$, which is the sum of several bounded functions, is bounded. Therefore, the sliding condition in (13) is ensured, which implies that the position tracking error tends to zero (and that the overall system is stable). In fact, from (13), $s$ will be bounded and converges to zero. Note that a drawback of the PEB method is that it does not guarantee a good transparency in term of force tracking. In order to improve the tracking performance, other schemes are proposed in the next sections.

2) Force error based (FEB)

A force-error-based, also called force-force, system is rarely used in the $2 \mathrm{CH}$ bilateral teleoperation system since two force sensors are required with no significant performance improvement. However, compared to the PEB architecture, this architecture can improve the force tracking performance. Fig. 3 shows the pneumatic-actuated FEB teleoperation system with a sliding mode control.

Consider the control law $u_{s}=u_{m}=\operatorname{sign}(s)$ where the sliding surface is defined as

$$
s=e=f_{h}-f_{e}
$$

where $e$ is the force tracking error. Using the same Lyapunov function as in (12), we need to show that the sliding condition (13) is satisfied.

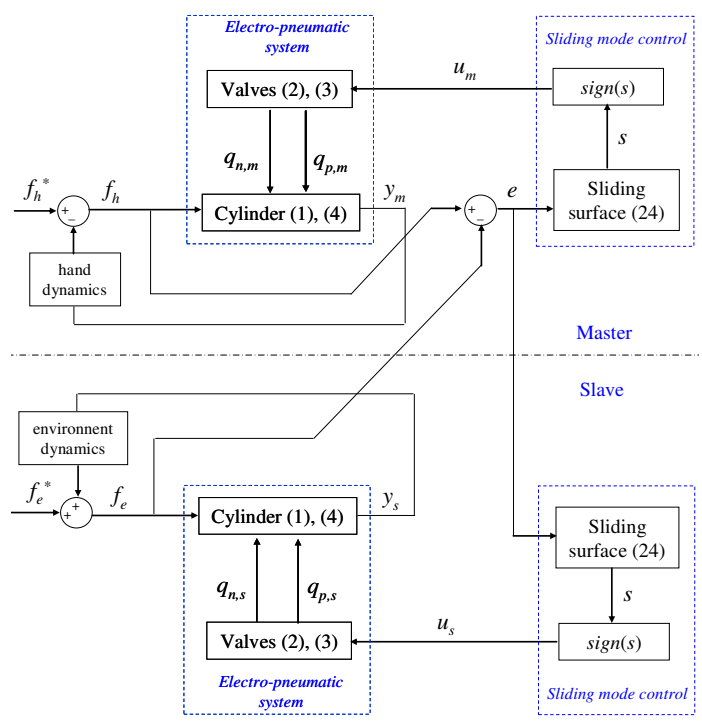

Fig. 3. Force-error-based approach with sliding mode control
From (7), we get

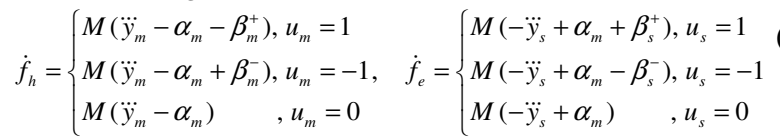

Case 1: $s>0$. In this case $u_{m}=u_{s}=1$. From (13), we need

$$
\dot{s}=\dot{f}_{h}-\dot{f}_{e}=M\left(\dddot{y}_{m}-\alpha_{m}-\beta_{m}^{+}+\dddot{y}_{s}-\alpha_{s}-\beta_{s}^{+}\right)<-\eta
$$

As it was demonstrated above, $\dddot{y}_{m}, \dddot{y}_{s}, \alpha_{m}$ and $\alpha_{s}$ are bounded. Thus, by choosing a valve with large orifice, $\beta_{m}^{+}$ and $\beta_{s}^{+}$can be made sufficiently large to satisfy (26).

Case 2: $s<0$. In this case, $u_{m}=u_{s}=-1$. We have

$$
\dot{s}=\dot{f}_{h}-\dot{f}_{e}=M\left(\dddot{y}_{m}-\alpha_{m}+\beta_{m}^{-}+\dddot{y}_{s}-\alpha_{s}+\beta_{s}^{-}\right)>\eta
$$

Similar to Case 1 , the stability of the system can be guaranteed by choosing a large enough value of $\beta_{m}^{-}$and $\beta_{s}^{-}$.

Consequently, the force tracking error converges to zero and the overall system is stable. However, the FEB method does not guarantee a good position tracking performance. In order to overcome the PEB and FEB architecture drawbacks, we use the scheme described in the following section.

\section{3) Direct force reflection (DFR)}

A direct-force-reflection, also called force-position, system has advantages over the position-position and forceforce architectures. The pneumatic-actuated DFR system with a sliding mode control is illustrated in Fig. 4.

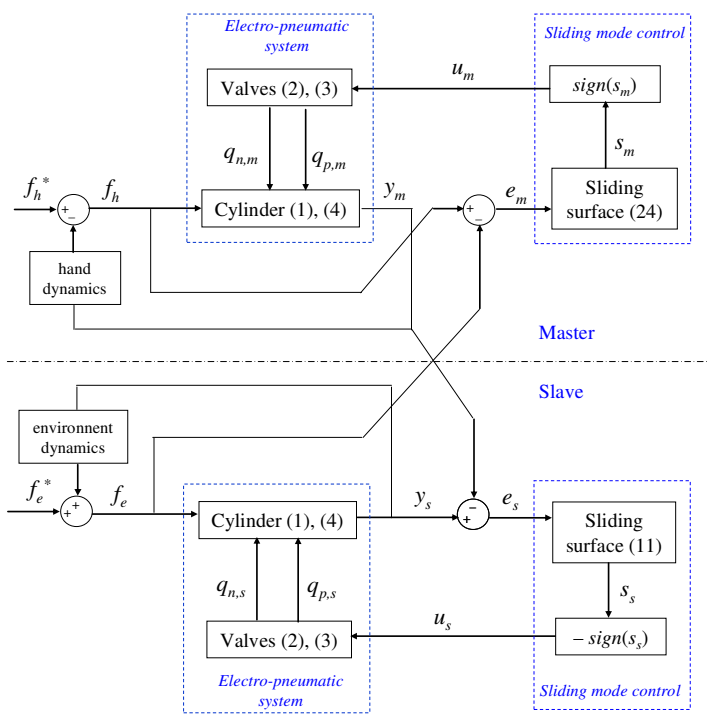

Fig. 4. Direct-force-reflection approach with sliding mode control

Here, $s_{m}$ and $s_{s}$ are the sliding surfaces for the master and slave systems, respectively; $e_{m}$ is the force tracking error of the master side; and $e_{s}$ is the position tracking error of the slave. This architecture involves two types of data transmission between the master and the slave: force from the slave to master and position from the master to the slave. Hence, the transparency is improved in terms of force and position tracking, compared to these previous methods. This statement will be justified later in the simulation. 
In this section, we use a Lyapunov function to prove the stability of the DFR system. First, we will show the stability of the force-controlled master manipulator. Afterwards, we will show the stability of the position-controlled slave manipulator. However, the stability of the overall system is difficult to show due to the different sliding surfaces for the master and the slave. Indeed, the following proves the stability of each manipulator, but not the stability of the overall teleoperation system.

\section{a) Force convergence in the closed-loop master system}

Since the desired force for the master robot, i.e., the slave/environment contact force $f_{e}$ is assumed to be bounded at the beginning, we need to show that $f_{h}$ converges to $f_{e}$ in a finite time. The sliding surface $s_{m}$ and the Lyapunov function $V_{m}$ are defined as in (24) and (12), respectively. The controller $u_{m}$ is chosen to be similar to the FEB system in section III.B.2.

Case 1: $s_{m}>0$. In this case, $u_{m}=1$. Using the expression of $\dot{f}_{h}$ in (25) and the definition of $s_{m}$ as in (24) we have

$$
\dot{s}_{m}=-\dot{f}_{e}+M \ddot{y}_{m}-M \alpha_{m}-M \beta_{m}^{+}
$$

To ensure the sliding condition (13), we need

$$
-\dot{f}_{e}+M \ddot{y}_{m}-M \alpha_{m}-M \beta_{m}^{+}<-\eta
$$

Similar to how it was demonstrated in section III.B.1, $\dot{f}_{e}, \dddot{y}_{m}$ and $\alpha_{m}$ can be shown to be bounded. Thus, the stability condition (29) is satisfied by choosing a large value of $\beta_{m}^{+}$.

Case 2: $s_{m}<0$. In this case, $u_{m}=-1$. From (24) and (25) we need

$$
-\dot{f}_{e}+M \ddot{y}_{m}-M \alpha_{m}+M \beta_{m}^{-}>\eta
$$

Similar to Case 1 , it is possible to choose a large enough value of $\beta_{m}^{-}$to ensure the stability of the master robot.

Consequently, the sliding surface (the force tracking error) tends to zero, i.e., $f_{h}$ tends towards $f_{e}$.

\section{b) Position convergence in the closed-loop slave system}

The sliding surface $s_{s}$ and the Lyapunov function $V_{s}$ are defined as in (11) and (12), respectively. The controllers $u_{s}$ is chosen to be similar to the PEB system in section III.B.1.

Case 1: $s_{s}>0$. In this case, $u_{s}=-1$. The sliding condition (13) is equivalent to

$$
\left(\dddot{y}_{s}-\dddot{y}_{m}\right)+2 \xi \omega \ddot{e}_{s}+\omega^{2} \dot{e}_{s}<-\eta
$$

Using the expression of $\dddot{y}_{s}$ in (16), we have

$$
\varphi-\beta_{s}^{-}<-\eta
$$

where $\varphi=\alpha_{s}-\dot{f}_{e} / M-\dddot{y}_{m}+2 \xi \omega \ddot{e}_{s}+\omega^{2} \dot{e}_{s}$

The straightforward reasoning described in section III.B.1 allows us to infer that $\varphi$ is bounded. Therefore, there exists a high value of $\beta_{s}^{-}$such as (31) is satisfied.

Case 2: $s_{s}<0$. In this case, $u_{s}=1$. Thus, we need

$$
\varphi+\beta_{s}^{+}>\eta
$$

This condition is achieved by choosing a large enough $\beta_{s}^{-}$.

Note that for both cases, the convergence of the sliding surface (and thus the position tracking errors) to zero is proved, so $x_{s}$ tends towards $x_{m}$.

As it will be confirmed in the next section, the DFR architecture provides a good transparency in terms of both position and force tracking.

\section{Simulations}

In this section, we verify the efficiency and performance of a teleoperation system using pneumatic actuators with on/off solenoid valves. The pneumatic and mechanical models of the master and the slave actuators are given by (1)-(3), and (6). The dynamic model of the operator's hand and the environment are presented in (22).

The $2 \mathrm{CH}$ bilateral teleoperation systems with three architectures PEB, FEB and DFR are implemented in Matlab/Simulink.

The initial values of the position and the velocity are set as $y_{m}(0)=y_{s}(0)=0.05$ and $\dot{y}_{m}(0)=\dot{y}_{s}(0)=0$. The initial pressures in the chambers are supposed to be equal to the atmospheric pressure. The environment is assumed to be passive $\left(f_{e}^{*}=0\right)$ and the operator's exogenous force

$$
f_{h}^{*}=15 \sin (t)+10 \sin (5 t)
$$

is chosen as the input signal of the teleoperation system.

For the PEB and DFR systems, the sliding surface of the position-controlled slave is defined in (11). To place the master and the slave closed-loop poles for fast responses without overshoot, the parameters $\xi=1$ and $\omega=100 \mathrm{rad} / \mathrm{s}$ are chosen.

Fig. 5 shows the master and the slave force and position tracking profiles for the PEB teleoperation system, where the slave is in contact with an soft environment whose dynamic is $Z_{e}=s^{2}+10 s+500$. Figures 6 and 7 illustrate the same profiles for the FEB and the DFR systems, respectively.

As illustrated in Fig. 5, the PEB system provides a good position tracking responses. However, the force response is deteriorated because no force sensor is used. On the other hand, the force tracking performance of the FEB system is improved, thanks to the knowledge of the force information. Nonetheless, the transparency is not perfect in term of position tracking. As it can be seen in Fig. 6, the slave's movement does not accurately track the master's movement.

The DFR system in Fig. 7 provides an improvement in position tracking response with respect to the FEB system. Moreover, it displays a superior force tracking performance compared to the PEB. Since the DFR uses the measurement of slave/environment contact forces, the feeling of contact motion is much more realistic. Therefore the performance of the teleoperation system is improved significantly by feeding the operator with the slave contact force. This result agrees with the previous theoretical work. 

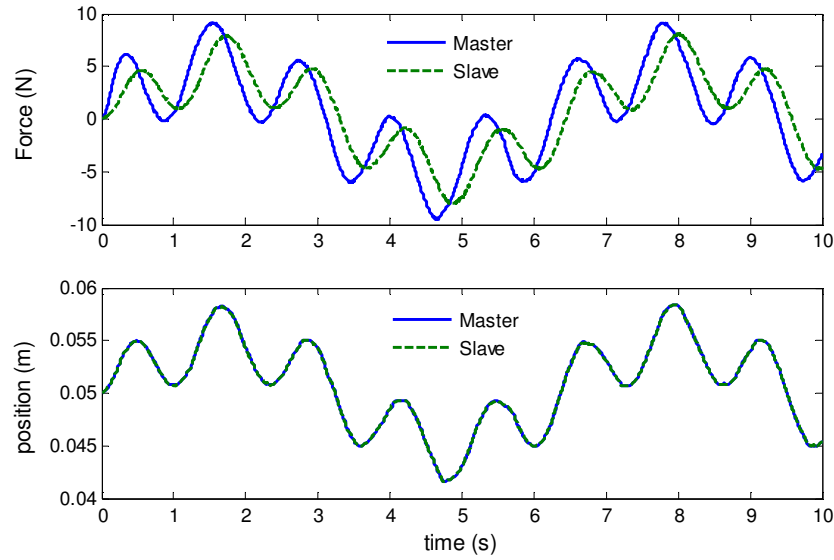

Fig. 5. Position and force profiles for the PEB teleoperation system
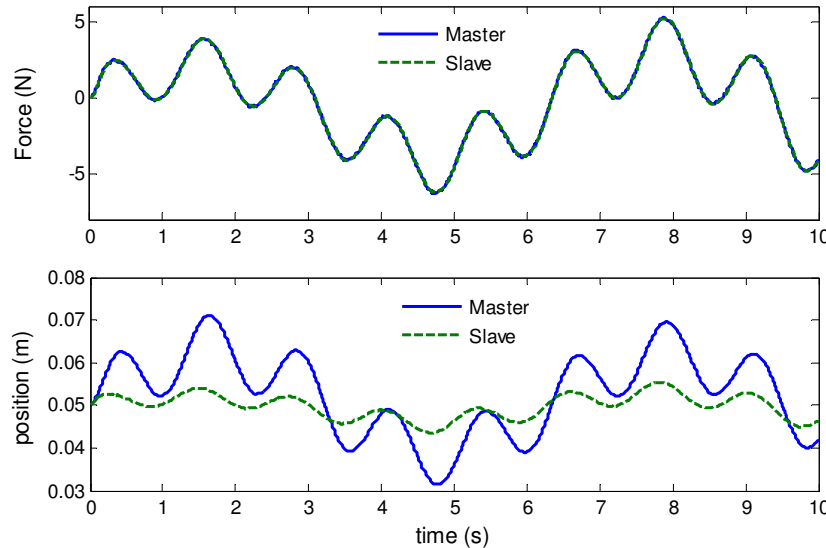

Fig. 6. Position and force profiles for the FEB teleoperation system
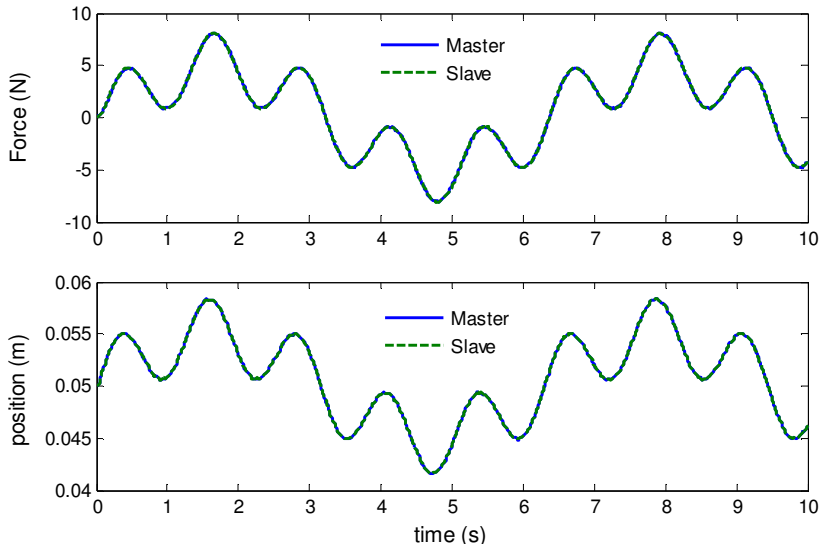

Fig. 7. Position and force profiles for the DFR teleoperation system

Among the three architectures, the DFR scheme seems to be a better choice to obtain a good transparency. Although the various teleoperation controllers have previously been compared from a performance perspective in the literature, this is the first study to show that it is possible to achieve stability and satisfactory performance using manipulators actuated by low-cost switching on/off valves.

\section{CONCLUSION}

In this paper, pneumatic actuators with inexpensive solenoid valves are chosen for the development of a masterslave teleoperation system. To efficiently control the switching on/off valves for haptic teleoperation system, sliding mode controllers are proposed and implemented in a realistic simulation. A comparison of the transparency and stability has been investigated between three control architectures (PEB, FEB and DFR) in a two-channel bilateral teleoperation system. The results show that better force and position tracking is obtained in the DFR architecture than in the PEB and FEB architectures. The simulation results are encouraging for future work aimed at implementing the proposed sliding bilateral control on an experimental testbed. Another aspect of this work is to incorporate sliding mode control in more complex teleoperation architectures (e.g., three or four-channel methods) in order to improve the performance of the teleoperation system.

\section{REFERENCES}

[1] P. F. Hokayem and M. W. Spong, "Bilateral teleoperation: An historical survey," Automatica, vol. 42, n. 12, p. 2035-2057, 2006.

[2] B. Hannaford, Scaling, impedance, and power flows in force reflecting teleoperation, American Mechanical Engineers, 1990.

[3] K. B. Shimoga, "A survey of perceptual feedback issues in dexterous telemanipulation: Part I. Finger force feedback," IEEE Virtual Reality International Symposium, p. 263-270, 1993.

[4] M. Ferre, M. Buss, R. Aracil, C. Melchiorri, and C. Balaguer, Advances in Telerobotics, Springer, vol. 31, 2007.

[5] M. Q. Le, M. T. Pham, M. Tavakoli, and R. Moreau, "Development of a hybrid control for a pneumatic teleoperation system using on/off solenoid valves," IEEE International Conference on Intelligent Robots and Systems, p. 5818-5823, 2010.

[6] N. Yu, W. Murr, A. Blickenstorfer, S. Kollias, and R. Riener, "An fMRI compatible haptic interface with pneumatic actuation," IEEE Conference on Rehabilitation Robotic, p. 714-720, 2008

[7] T. Noritsugu, "Pulse-width modulated feedback force control of a pneumatically powered robot hand," Proceedings of International Symposium of Fluid Control and Measurement, p. 47-52, 1985.

[8] M. C. Shih and M. Ma, "Position control of a pneumatic rodless cylinder using fuzzy PWM control method," Mechatronics, vol. 41, n. 2, p. 241-253, 1998.

[9] M. Taghizadeh, A. Ghaffari, and F. Najafi, "Improving dynamic performances of PWM-driven servo-pneumatic systems via a novel pneumatic circuit," ISA Transactions, vol. 48, n., p. 512-518, 2009.

[10] M. Q. Le, M. T. Pham, R. Moreau, and T. Redarce, "Comparison of a PWM and a Hybrid Force Control for a Pneumatic Actuator Using On/off Solenoid Valves," IEEE International Conference on Advanced Intelligent Mechatronics, p. 1146-1151, 2010.

[11] M. Q. Le, M. T. Pham, R. Moreau, and T. Redarce, "Transparency of a pneumatic teleoperation system using on/off solenoid valves," IEEE International Conference on Robot and Human Communication, p. 15-20, 2010.

[12] T. Nguyen, J. Leavitt, F. Jabbari, and J. E. Bobrow, "Accurate sliding-mode control of pneumatic systems using low-cost solenoid valves," IEEE/ASME Transactions on mechatronics, vol. 12, n. 2, p. 216-219, 2007.

[13] R. Blackburn, Shearer, Fluid Power Control. John Wiley and Sons, Inc, 1960.

[14] D. McCloy, "Discharge characteristics of servo valve orifices," Fluid International Conference, p. 43-50, 1968. 